\title{
Calpains Induce Egg Production Through Mirnas Secretion in Extracellular Vesicles Released From Paired Adult Worms of Schistosoma Japonicum
}

\author{
Takashi Kumagai ( $\nabla$ tkuma.vip@tmd.ac.jp ) \\ Tokyo Medical and Dental University \\ Rieko Shimogawara \\ Tokyo Medical and Dental University \\ Koichiro Ichimura \\ Juntendo University School of Medicine \\ Shiroh Iwanaga \\ Osaka University
}

\section{Research Article}

Keywords: Extracellular vesicles (EVs), miRNAs, egg production, calpain inhibitor

Posted Date: August 10th, 2021

DOI: https://doi.org/10.21203/rs.3.rs-783057/v1

License: (c) (i) This work is licensed under a Creative Commons Attribution 4.0 International License. Read Full License 


\section{Abstract}

Extracellular vesicles (EVs) have been reported to be secreted from Schistosoma japonicum at all developmental stages. However, the reproduction and communication mechanisms between the paired adults through the EVs in dioecious Trematoda have not been reported. In this study, EVs containing many exosome-like vesicles and microvesicles were observed in the supernatants of paired adults cultured in vitro, and abundant selected miRNAs were contained in them. In particular, the female-specific miR-bantam was present only in vesicles and was hardly secreted outside the vesicles. In this study, we found that male-female pairing induced secretion of miR-3479 and miR-bantam in EVs, but not of male-specific miR-61. Furthermore, ingestion of mouse erythrocytes also increased the production of miRNAs in paired adult and single female worms. Vesicles were found in the teguments of females treated with erythrocytes under electron microscopy. After the paired worms were treated with several inhibitors against the secretion of EVs, only calpain inhibitor (calpeptin) significantly reduced the amount of miRNA in EVs. Furthermore, the worms treated with only calpeptin inhibited egg production in vitro. Together, these results indicate that qualitative miRNA production through EVs regulated by calpain plays a role in egg production in S. japonicum.

\section{Introduction}

Schistosomes are parasitic helminths distributed in tropical and subtropical regions, and schistosomiasis, known as a neglected tropical disease (NTD), with more than 250 million infected people worldwide and more than 779 million people living in areas at risk of infection. ${ }^{1}$. The genus Schistosoma is characterized as a dioecism different from the other hermaphrodite flukes in the phylum Platyhelminthes. Schistosomes are parasites in the blood veins of their mammalian hosts, ingesting red blood cells for nutrition and egg laying. Egg laying causes pathological changes in the final host, leading to egg granulomas and fibrosis of the liver and intestines. Although egg laying is directly linked to symptoms and has become a target for drug and vaccine development, the mechanism of sexual maturation required to induce egg laying is not well understood.

The most important event in the life cycle of the schistosome is the laying of eggs and the production of offspring for the next generation. A female worm cannot sexually mature and lay eggs unless she is paired with a male worm ${ }^{2-5}$. On the other hand, the uptake of red blood cells by paired adults is also necessary for sexual maturation and egg laying ${ }^{6,7}$. The sex-related genes and proteins were up- or down-regulated in $S$. mansoni and S. japonicum before and after pairing ${ }^{4,8-10}$. Furthermore, elevated miRNAs regulating mRNA expression were also observed in $S$. japonicum during the pairing phase ${ }^{11-13}$. Cai et al. reported that the expression of a set of miRNAs, sja-miR-7-5p, sjamiR-61, sja-miR-219-5p, sja-miR-125a, sja-miR-125b, sja-miR-124-3p, and sja-miR-1 were dominant in male worms, whereas sja-miR-bantam, sja-miR-71b-5p, sja-miR-3479-5p, and sja-Novel-23-5p were predominantly found in female parasites of S. japonicum ${ }^{11}$. Moreover, Zhu et al. reported that 14 miRNAs were enriched in male worms, whereas four miRNAs were predominantly found in females ${ }^{13}$. In common, miR-bantam, a female-specific miRNA, is drastically upregulated after pairing ${ }^{12-14}$. It has been reported that antisense RNA against miR-bantam inhibits ovary formation ${ }^{13}$. Moreover, miR-bantam and miR-3479, miR-10, and miR-277 have been reported to be secreted from the parasite in the sera of infected animals ${ }^{15,16}$. Those miRNAs are the main components of the extracellular vesicles (EVs) derived from S. japonicum ${ }^{17,18}$.

Recently, EVs, including exosomes, microvesicles, and apoptotic bodies, have been shown to function in communication between cells ${ }^{19-21}$. Exosomes within the size range of 30-150 nm were derived from the multivesicular bodies through the endosomal pathway, although microvesicles with a wide size range of 100-1000 nm were directly formed from cell membranes through budding. In particular, EVs contain various nucleic acid 
compositions, including mRNAs, miRNAs, and DNA fragments, in addition to proteins and lipids. These nucleic acids in EVs can function in recipient cells through EV cargo. In reports on schistosomes, mixed types of EVs were found in eggs, schistosomula, and adults at the stages of schistosomes ${ }^{22-26}$. In particular, in S. japonicum and Fasciola hepatica, the EVs derived from the parasites are transferred to host cells and regulate immune responses in hostparasite interactions ${ }^{17,27-31}$. In addition, there was the most recent report on the proteins and miRNAs contained in the extracellular vesicles in each male and female. It was reported that miR-750 was enriched in the extracellular vesicles of females and that ovary formation and egg laying of paired insect bodies treated with miR-750 antisense was suppressed ${ }^{26}$. Thus, it was shown that miRNAs in extracellular vesicles may be involved in egg production. However, the communication between males and females of parasites through the EVs is unknown.

In this study, to investigate how EV secretion is induced, EVs secreted from paired adults were extracted. We observed that the pairing of males and females triggered miRNAs, particularly female-specific miR-bantam secretion via EVs. Furthermore, ingestion of red blood cells by paired worms significantly increased miRNA production. To confirm how EVs were secreted and formed in the worms, the paired adult worms were treated with several inhibitors of EV production. The inhibitor of calpain, a calcium-dependent cysteine protease, strongly reduced the production of miRNAs in EVs and reduced egg production in paired worms. Our results will provide new insights into the formation and roles of EVs in schistosomes.

\section{Methods}

\section{Ethics statement}

All animal experiments were performed under the section of Environmental Parasitology, Tokyo Med. \& Dent. Univ (TMDU). All animal experiments were approved by the Institutional Animal Care and Use Committee of Tokyo Medical and Dental University (A2021-131A).

\section{Animal and Parasite}

To maintain the life cycle of Schistosoma japonicum, ICR mice (5weeks old) from SLC (Hamamatsu, Japan) and the intermediate host, Oncomelania hupensis nosophora (Yamanashi strain), were infected as previously described ${ }^{55}$. Snails collected from the field were infected with five to seven miracidia each. They were kept in an thermostatic chamber at $28^{\circ} \mathrm{C}$ for over four months. The infected shells were placed between glass slides to crush the shells and release the cercariae inside. The mice anesthetized by peritoneal administration of sodium pentobarbital in saline $(40 \mathrm{mg} / \mathrm{kg})$ have their abdomens shaved and were infected with a cover glass to which 50 cercariae were attached.

The infected animals were kept in a controlled temperature and humidity environment with a 12:12 h light: dark cycle. The mice had free access to water and food in the facilities approved by the Institutional Animal Care and Use Committee of Tokyo Medical and Dental University (2010002C2). Humane endpoints were applied when an evidence of severe pain, excessive distress, suffering or an impending death was observable in any of the animals, which were then euthanized. All mice were euthanized at the end of the experiment by intraperitoneal injection of sodium pentobarbital in saline solution $(100 \mathrm{mg} / \mathrm{kg})$. The status of all mice was checked daily using a composite score including vitality, secretions, fur quality, mobility, dyspnea, ascites, neurological signs, and ability to ingest water or food. All methods were carried out in accordance with relevant guidelines and regulations. All animal handling and methods complied with the ARRIVE guidelines. 
Adult worms were collected by perfusion from euthanized mice at seven weeks post-infection. They were placed in a conditioned medium consisting of RPMI1640 containing 10\% exosome-depleted FBS (Thermo Fisher Science, USA), penicillin, streptomycin, L-glutamine (Thermo Fisher Science). After 24 h, all worms were washed with the conditional medium three times, and the surviving worms were used for experiments.

\section{Extracellular vesicles (EVs) secreted from parasites}

To collect EVs, adult worms (three pairs) were cultured in a conditional medium for a week at $37^{\circ} \mathrm{C}$ in a $\mathrm{CO}_{2}$ incubator. For TEM analysis, the supernatant was subjected to ultracentrifugation at 100,000 $\times \mathrm{g}$ for two hours and washed twice with PBS. Alternatively, for RNA extraction, the supernatant was treated with the Total Exosome Isolation Reagent (Thermo Fisher Scientific). The pellet was dissolved in TRI Reagent (MRC, USA). Furthermore, to check whether the EVs were vesicles containing phosphatidylserine in the membrane, the MagCapture ${ }^{\mathrm{TM}}$ Exosome Isolation Kit PS (WAKO, Japan) was used to purify the vesicles. In brief, the supernatant from the worms (three pairs/well) cultured for three days was used as a sample for purification using this kit. RNA extraction was performed by adding TRI Reagent directly to the beads binding to the EVs after washing. On the other hand, Total Exosome Isolation Reagent was added to the flow-through fractions and RNA not contained in EVs was extracted using the same procedure as above.

\section{Cultured paired worms and treatment with RBCs and inhibitory experiment of EV production by inhibitors}

The paired adult worms were directly picked up, and single-sex worms detached naturally through incubation for $24 \mathrm{~h}$ were also used as a single sex worm. All worms were placed into a 12 well-culture plastic plate in conditional medium. For treatment with erythrocytes, RBCs were prepared from the whole blood of euthanized ICR mice ( 5 weeks old). The cells were collected from the RBC fraction after centrifugation at $3,000 \times \mathrm{g}$ and washed with the conditional medium three times. Furthermore, ghost RBCs were prepared after puncturing and reconstruction of RBCs as a standard method. Adult worms (three pairs of worms or three worms per well; 5 wells/group) were cultured with or without RBCs or ghost RBCs $\left(10^{8}\right.$ cells) in a $2 \mathrm{ml}$ conditional medium for a week at $37^{\circ} \mathrm{C}$ in a $\mathrm{CO}_{2}$ incubator. After culture, all worms were confirmed to survive, and the supernatant was collected for EV preparation. All worms were collected for RNA extraction and the female worms cultured with RBC were collected for transmission electron microscopy (TEM). For the inhibitor test, three paired worms were treated with EV inhibitors suspended in DMSO, and then mouse RBCs were added after an hour. The appropriate concentration of each inhibitor was based on data from previous studies (10 $\mu \mathrm{M}$ Y27632, $5 \mu \mathrm{M}$ Manumycin A, $80 \mu \mathrm{M}$ Calpeptin, $10 \mu \mathrm{M}$ GW4869) ${ }^{33}$. After three days, the supernatants were collected from each well for RNA extraction from EVs, and all worms were removed for egg counting. The eggs were treated with $4 \% \mathrm{KOH}$ at $37^{\circ} \mathrm{C}$ overnight, and all eggs were counted under a microscope.

\section{Transmission electron microscopy (TEM) analysis}

The isolated EVs were loaded and negatively stained with $1 \%(\mathrm{w} / \mathrm{v})$ uranyl acetate solution on copper grids (400 mesh) covered with formvar film. These specimens were observed using TEM at $75 \mathrm{kV}(\mathrm{H}-7100$, Hitachi, Tokyo, Japan). TEM using adult worms was performed as previously described ${ }^{56}$. In brief, the adult single female worms after ingestion of the erythrocytes were fixed in $2.5 \%$ glutaraldehyde in $0.1 \mathrm{M}$ phosphate buffer for $24 \mathrm{~h}$. The fixed worms were successively immersed in $0.5 \%$ osmium tetroxide $\left(\mathrm{OsO}_{4}\right)$ in $0.1 \mathrm{M}$ phosphate buffer for an hour, $2 \%$ lowmolecular-weight tannic acid (LMW-TA, Electron Microscopy Sciences, Hatfield, PA) in $0.05 \mathrm{M}$ maleate buffer for four hours, and $1 \%$ uranyl acetate in $0.05 \mathrm{M}$ maleate buffer for three hours. The worms were then dehydrated with a graded series of ethanol and embedded in Oken Epok 812 epoxy resin (Oken-shoji, Tokyo, Japan). Ultrathin sections (90-100 nm thickness) were produced using an ultra $45^{\circ}$ diamond knife (Diatome, Biel, Switzerland) and transferred 
to 50-mesh copper grids coated with a Formvar membrane. Ultrathin sections stained with lead citrate and uranyl acetate were digitally photographed using an H-7100 transmission electron microscope (Hitachi High-Technologies, Tokyo) equipped with a C4742-95 CCD camera (Hamamatsu Photonics, Shizuoka, Japan).

\section{RNA extractions and cDNA synthesis of miRNA}

RNA was extracted from EVs using Direct-zol ${ }^{T M}$ RNA Kits (Zymo Research, USA). The quality of the extracted RNA was evaluated using a Bioanalyzer (Agilent, USA), and the concentration of total RNA was also measured. cDNA synthesis for miRNA detection was performed using a Simple miRNA Detection Kit (Biodynamics, Japan) or Mir- $\mathrm{X}^{\mathrm{TM}}$ miRNA First-Strand Synthesis Kit (Takara Bio Inc., Japan).

\section{miRNA-seq library and sequencing}

The miRNA-seq was performed using total RNA samples by contact analysis (GNAS, Japan). In brief, miRNA-seq libraries were prepared from 50 ng of total RNA using the TruSeq RNA Library Preparation Kit (Illumina, UK). All samples were processed with three biological replicates from five worm groups representing one biological replicate. Libraries were multiplexed and sequenced on an Illumina HiSeq 2500 with 50 bp paired-5'end reads. The sequence counts were detected as expression values and annotations were determined by inputting them into the miRBase of the $S$. japonicum. The value of each expression value when the total count was set to one million was used as the data in each sample. The top 20 annotated miRNAs were enumerated by averaging each of the three types of data.

\section{Quantitative real-time PCR (qRT-PCR) analysis}

cDNA prepared from miRNAs from EVs was used for qRT-PCR. Briefly, miRNA expression of EVs was analyzed using universal primers and miRNA-specific forward primers (Sup. S1 Table) in a mixture of the KAPA SYBR Fast qPCR kit (Kapa Biosystems, South Africa). The reaction was performed using the LightCycler480 system (Roche, USA) at $95^{\circ} \mathrm{C}$ for $2 \mathrm{~min}$, followed by 45 cycles of $95^{\circ} \mathrm{C}$ for $10 \mathrm{~s}, 60^{\circ} \mathrm{C}$ for $10 \mathrm{~s}$, and $72^{\circ} \mathrm{C}$ for $1 \mathrm{~s}$. After amplification, the reaction was performed at $95^{\circ} \mathrm{C}$ for $5 \mathrm{~s}, 60^{\circ} \mathrm{C}$ for $1 \mathrm{~min}$, and then gradually heated to $95^{\circ} \mathrm{C}$ for melting curve analysis. In the expression analysis of miRNAs, the concentration of EVs RNAs recovered in another experiment was measured by the bioanalyzer, and a standard line for analysis was drawn using this as the standard concentration. each miRNA in EVs was measured by the absolute quantification method against the standard line. In each graph, the absolute quantitative value of each miRNA was expressed as a relative value to the value of the control group to be compared.

\section{Statistical analysis}

Statistical differences between the two groups were assessed using an unpaired two-tailed t-test and were considered significant at $p<0.05$. In the miRNA expression analysis, statistical analysis was performed based on the miRNA absolute quantitative values in EVs obtained from three to five groups of cultured adult worms and compared between two groups. In the inhibitor experiment, statistical analysis was performed by comparing between the DMSO-treated groups and each treated group.

\section{Results}

\section{The pairing between males and females triggered the secretion of selected miRNAs in the EVs.}

In previous study, although the secretion of extracellular vesicles (EVs) has been confirmed at each developmental stage of $S$. japonicum, there are few reports on its induction or formation. In general, as EVs are used for cell-cell communication, they are considered to be actively used for male and female communication in living schistosomes, 
and in this study, we focused on the relationships between the pairing and secretion of EVs. TEM examination of EVs collected from cultured paired adults revealed that the isolated EVs contained heterogeneous vesicles of less than $100 \mathrm{~nm}$, exosome-like vesicles, and microvesicles, which were not only abundant but also contained some protein complexes (Fig. 1). Next, miRNA-seq was performed using EVs from the supernatant fluid of paired worms collected under three independent cultures. After sequencing three different samples, the total number of reads was 43912068, 44647592 , and $42,775,333$, respectively. The annotated miRNAs were determined by entering the detected sequences into the $S$. japonicum database in miRBase (Table 1). miRNA-seq results showed that EVs secreted from paired adults contained miRNAs similar to those reported in previous studies. ${ }^{17,18,24}$. In particular, some sex-biased miRNAs, miR-bantam(female), miR-61 (male), let-7(male) ${ }^{13,32}$, and miRNAs detected in the serum of the infected animals (miR-3479, miR-10, miR-bantam, miR-277, miR-71, let-7) ${ }^{15,16}$, were also observed.

Focusing on several miRNAs, the amount of both miR-bantam and miR-3479 increased significantly in the EVs of the paired worms than in those of single-sex worms (Fig 2). The increase rate of both miRNAs in paired worms was about three to four times, which was larger than the sum of the expression levels of single males and females. In particular, miR-bantam, which is specifically expressed in females, was only expressed in EVs from adult females. This suggests that miR-bantam secretion from females was very strongly induced by pairing. On the other hand, miR61 , whose expression is specifically observed in males, was not induced by pairing. It has been reported that miR-61 is a miRNA whose expression increases with growth from the schistosome stage and, unlike miR-bantam, does not show a dramatic increase in expression by pairing ${ }^{13}$. In other words, it was considered that the qualitative difference in miRNA produced in EVs was induced by pairing. Although the expression level of miR-36, which is not detected in the serum of infected animals, was high in miRNA-seq, the amount secreted by pairing was only the sum of the amounts secreted by males and females, and no induction of expression was observed. In conclusion, pairing had a synergistic effect on the induction of production of selected miRNAs such as miR-bantam and miR-3479.

To confirm whether these qualitative differences are due to the secreted form (vesicles or protein complexes), we selected only vesicles using phosphatidylserine (PS)-targeted column and measured their miRNA expression. Two miRNAs (miR-bantam and miR-36) were explicitly expressed in the vesicles (S1 Fig). However, miR-3479 and miR-61 were not only packaged in vesicles, but also formed complexes with proteins outside the EVs. Taken together, these data suggest that the qualitative secretion of miRNA is not dependent on the type of vesicle. However, the femalespecific miR-bantam was present only in vesicles, confirming that it was produced in large quantities through vesicles from female worms after pairing.

\section{Ingestion of erythrocytes by adults increased the amount of miRNA production.}

It has also been reported that ingestion of erythrocytes by schistosomes induces sexual maturation and egg deposition in female worms ${ }^{6,7}$. We measured the miRNA in the EVs after adding mouse erythrocytes to paired worms or single-sex worms. As a result, both miR-bantam and miR-3479 production were increased in the EVs from paired worms after ingestion of erythrocytes (Fig 3A, 3B). However, the production levels of the other miRNAs (miR-61 and miR-36) did not change (S2 Fig). These results confirmed that the secretion of miRNAs selectively induced by pairing was further increased by the ingestion of red blood cells. It has also been reported that the heme proteins in erythrocytes play an essential role in egg production ${ }^{6}$. Therefore, we administered ghost erythrocytes, which consist only of erythrocyte membranes, to paired worms. No differences in miRNA expression levels were observed in either 
group (S3 Fig). These data indicate that the factors that induce miRNA production in EVs are the cytoplasm of erythrocytes, such as heme proteins. Furthermore, the secretion of miRNAs was significantly increased in single cultures of female worms containing erythrocytes (Fig 3A, 3B). This result showed that female worms play a central role in miRNA secretion via EVs in the paired state. In addition, electron microscopy of the body surface of the female worm treated with erythrocytes showed clear vesicles in the tegument of the tubular structure (Fig 3C). These results suggest that when red blood cells are ingested during pairing, EVs produced by the female worms transmit miRNAs through the tegument in contact with the surface of the male worms.

\section{Reduction of miRNA expression and egg production treated with inhibitors against EV secretion}

In general, EVs are known to be secreted by various pathways depending on the type of vesicle and the location of vesicle formation ${ }^{19,33-35}$. However, no studies have been conducted on the secretory mechanism of EVs in schistosomes. In the present study, to confirm the mechanism of EV-mediated miRNA secretion induced by pairing with erythrocyte ingestion, we treated paired adults with chemical inhibitors that have already been reported to suppress EV production ${ }^{33}$. Known inhibitors of exosome secretion include manumycin A, which inhibits the ESCRTdependent pathway to multi-vesicular bodies, and GW4869, a neutral sphingomyelinase (nSMase) inhibitor that inhibits the ESCRT-independent pathway, is known ${ }^{34,36}$. On the other hand, calpeptin, an inhibitor of calpain, a calcium-dependent neutral cysteine protease, is also known to be involved in microvesicle shedding ${ }^{37}$. In addition, Y27632 also inhibits the production of microvesicles through Rho-associated protein kinase (ROCK), which are serine-threonine kinases involved in cytoskeleton re-organization. ROCK regulates microvesicle shedding and apoptotic blending via the myosin light chain ${ }^{38}$. These inhibitors were used to induce the secretion of EVs by treating paired adult worms with effective concentrations and then culturing them with mouse erythrocytes. After 3 days of incubation, EVs collected from the supernatant were extracted and the expression levels of miRNAs were measured. As a result, only calpain inhibitor (calpeptin) reduced all selected miRNAs in the collected EVs (Figs 4A-C). In addition, two exosome-inhibitors (manumycin A and GW4869) also reduced miR-bantam secretion, although it was not as effective as calpeptin. These results indicate that calpain plays a predominant role in the miRNA secretion of EVs in schistosomes. On the other hand, only calpeptin treatment inhibited the egg production of paired adults (Fig 4D). These results showed that calpain affects miRNA production in EVs from paired adult worms, and calpain may induce sex maturation and egg production via EVs.

\section{Discussion}

Previous studies have confirmed the secretion of extracellular vesicles (EVs) from each developmental stage of schistosome 22-26,39,40, but little is known about the mechanisms involved in vesicle formation and secretion. In the present study, we observed EV induction after the pairing and ingestion of erythrocytes. Originally, pairing and erythrocyte ingestion are known to induce sex maturation in adult worms and egg deposition in female worms ${ }^{2,5,6}$. A previous report by proteomic analysis in S. mansoni showed that paired females increased vesicle transport-related molecules ${ }^{9}$. In addition, an increase in RNA transporter-related and ribosomal proteins was confirmed in $S$. japonicum ${ }^{3}$. These reports may support the observation in this study that pairing induces EV formation and miRNA transport. Furthermore, our study confirmed that EV secretion is increased when paired females ingest erythrocytes, but until now there is little information on the analysis of the transcriptome and proteome induced by erythrocyte ingestion. Our results suggest that it is the female worms that caused the increase in EVs of the paired worms. In fact, electron microscopy revealed the presence of vesicles in the tegument of the female worm that ingested the red 
blood cells. This indicates that female worms may be transmitting reproductive information via EVs through contact with male worms. Direct contact with male worms is necessary for female sexual development $2,41,42$, and interestingly, vitelline maturation occurs only in regions in direct contact with the male segment ${ }^{5,43}$. These studies showed that females paired with males initiate signals that induce local vitelline maturation in females. The presence of EVs on the female tegument suggests that EVs play a role in direct signal transduction in local contact between females and males.

In addition to a quantitative increase in EV miRNA secretion by pairing, the qualitative selection was observed. This represents the difference in miRNAs that are packed into EVs during pairing. Female-specific miR-bantam has been reported to have a rapid increase in expression after the pairing stage, while other miRNAs that are similarly upregulated (miR-1989, miR-31, miR-2c) are not present in EVs ${ }^{13}$. Therefore, the qualitative selection may be performed for packing into EVs in the pairing of schistosomes. It was also observed that miR-bantam and miR-3479 increased by pairing, but each miRNA was packed in different types of EVs. In particular, miR-bantam was mostly identified in vesicles, while miR-3479 was thought to be secreted by forming a complex with RNA proteins as well as vesicles. From this, it was considered that the secretion of miRNA by schistosomes involves multiple routes. In addition, miR-bantam is specific to females and localizes only in the vesicles contained after pairing, suggesting that it is an ideal EV marker for the reproductive phase. In this study, monitoring miR-bantam was also considered to be an optimal marker for EV detection and a reference miRNA for the analysis of EV-mediated male-female communication. The detection of miR-bantam has also been confirmed in blood samples from experimental infections ${ }^{15,16,44}$ and it may also be an effective marker for monitoring active infections via EV secretion.

Our results confirm that EVs are induced in schistosomes by erythrocyte ingestion and female/male pairing, but there is little information on the mechanism by which EVs are formed and secreted. Based on the reports of EVs inhibitors that inhibit the secretion of each vesicle ${ }^{33}$, we conducted experiments to inhibit EV secretion in schistosomes. As a result, only calpeptin suppressed the secretion of all tested miRNAs via EVs. This result is the first evidence that calpeptin is involved in miRNA secretion in EV secretion studies in schistosomes. Furthermore, this result suggested that most of the miRNAs secreted from adults of schistosome may be related to microvesicles, because calpeptin is known to inhibit the shedding of microvesicles promoted by calpain activity on cytoskeleton remodeling ${ }^{33,37}$. However, the decreased secretion of not only miR-bantam, which is only found in vesicles, but also other miRNAs suggested that calpain may be involved in the secretion mechanism of miRNAs itself, because it has been reported that calpain is also abundant in EVs and is secreted by worms $25,39,45,46$. In fact, in S. mekongi, calpain was detected inside the membrane-bound vesicles in the female tegument under electron microscopy ${ }^{46}$. Furthermore, it has been reported that extracellular tegumental calpains inhibit the blood clot formed on the surface of the parasite by cleaving fibronectin ${ }^{46,47}$. Taken together, these reports suggest that calpain may be directly involved in the secretion of miRNA via EVs and may function as one of the components of EVs carried from females to males. In contrast, only miR-bantam secretion was reduced by the exosome inhibitors manumycin A and GW4869. It was considered that a part of miR-bantam was released by exosome-like vesicles. A recent study in Fasciola hepatica, which is closely related to schistosoma, reported that small vesicles called $120 \mathrm{k}$ vesicles were released by nSMase, and the secretion was inhibited by GW4869 ${ }^{48}$. This study supports the hypothesis that miR-bantam secretion is partially suppressed by exosome inhibition in schistosomes.

In addition to the sufficient inhibitory effects of the inhibitors on the secretion of several miRNAs, the present study also revealed that calpeptin inhibited egg laying in female worms. Previous studies have already reported the effect of calpain vaccine in reducing the number of eggs in schistosomes ${ }^{49-51}$. Since the egg-laying inhibition effect seen 
with the calpain vaccine is also seen with other vaccines ${ }^{52}$, we consider that this effect may be due to an immune response induced by the vaccine. However, the present study confirms that calpain inhibitors have a direct inhibitory effect on the fecundity of schistosomes. In addition, several studies have reported that calpain localizes to the surface of adult worms, not to the female reproductive organs $46,53,54$. This means that calpain does not function directly in the reproductive organs, suggesting the involvement of calpain in the secretion of EVs from the tegument and the induction of egg laying. On the other hand, there is a report that miR-bantam is involved in egg-laying induction by being taken up by host cells ${ }^{18}$. In this report, it was observed that uptake of EV miRNAs including miRbantam into host cells increased macrophage proliferation and TNF-a production, indicating that TNF-a production in the host is also involved in egg production of $S$. japonicum. This could be that the decreased expression of miRbantam caused the suppression of egg laying. However, in the present experiment, we confirmed that the production of miR-bantam increased only when red blood cells (without leukocytes) were added in vitro. In other words, egglaying inhibition was induced in the absence of immune cell effects, suggesting that calpeptin had a direct effect on the adult worms. Since it was confirmed that calpain inhibitors affected both egg production and miR-bantam secretion, the mechanism of egg production was directly related to the contribution of calpain and the associated EVmediated secretion of miRNAs such as miR-bantam.

In conclusion, the secretion of miRNAs via EVs was increased by erythrocyte pairing and ingestion. In particular, the secretion of miR-bantam via EVs in female worm bodies was remarkable, and vesicles were confirmed by TEM to be secreted from the worm surface. This increase in EVs was due to internal components (e.g., heme proteins) rather than erythrocyte membranes. Furthermore, we found that calpain inhibitors suppressed EV-mediated secretion of miRNAs. In addition, calpain inhibitor suppressed egg production in adults, suggesting that EVs transported from females to males affect sexual maturation and reproduction. Future studies are expected to develop a direct link between EV production, calpains, and egg production.

\section{References}

1. McManus, D. P. et al. Schistosomiasis. Nat. Rev. Dis. Prim. 4, 1-19 (2018).

2. Kunz, W. Schistosome male-female interaction: induction of germ-cell differentiation. Trends Parasitol. 17, 227-231 (2001).

3. Sun, J., Li, C. \& Wang, S. The up-regulation of ribosomal proteins further regulates protein expression profile in female Schistosoma japonicum after pairing. PLoS One 10, (2015).

4. Cheng, G. et al. Proteomic analysis of differentially expressed proteins between the male and female worm of Schistosoma japonicum after pairing. Proteomics 5, 511-21 (2005).

5. Wang, J., Chen, R. \& Collins III, J. J. Systematically improved in vitro culture conditions reveal new insights into the reproductive biology of the human parasite Schistosoma mansoni. PLOS Biol. 17, e3000254 (2019).

6. Toh, S. Q., Gobert, G. N., Malagón Martínez, D. \& Jones, M. K. Haem uptake is essential for egg production in the haematophagous blood fluke of humans, Schistosoma mansoni. FEBS J. 282, 3632-3646 (2015).

7. Wang, J. et al. Intake of Erythrocytes Required for Reproductive Development of Female Schistosoma japonicum. PLoS One 10, e0126822 (2015).

8. Sun, J. et al. Transcriptome profilings of female Schistosoma japonicum reveal significant differential expression of genes after pairing. Parasitol. Res. 113, 881-892 (2014).

9. Fitzpatrick, J. M. \& Hoffmann, K. F. Dioecious Schistosoma mansoni express divergent gene repertoires regulated by pairing. Int. J. Parasitol. 36, 1081-1089 (2006). 
10. GREVELDING, C. G., SOMMER, G. \& KUNZ, W. Female-specific gene expression in Schistosoma mansoni is regulated by pairing. Parasitology 115, S0031182097001728 (1997).

11. Cai, P. et al. Profiles of Small Non-Coding RNAs in Schistosoma japonicum during Development. PLoS Negl. Trop. Dis. 5, e1256 (2011).

12. Sun, J., Wang, S., Li, C., Ren, Y. \& Wang, J. Novel expression profiles of microRNAs suggest that specific miRNAs regulate gene expression for the sexual maturation of female Schistosoma japonicum after pairing. Parasit. Vectors 7, 177 (2014).

13. Zhu, L. et al. MicroRNAs Are Involved in the Regulation of Ovary Development in the Pathogenic Blood Fluke Schistosoma japonicum. PLoS Pathog. 12, (2016).

14. Han, Y. et al. Differential expression of microRNA between normally developed and underdeveloped female worms of Schistosoma japonicum. Vet. Res. 51, 126 (2020).

15. CHENG, G., LUO, R., HU, C., CAO, J. \& JIN, Y. Deep sequencing-based identification of pathogen-specific microRNAs in the plasma of rabbits infected with Schistosoma japonicum. Parasitology 140, 1751-1761 (2013).

16. Hoy, A. M. et al. Parasite-Derived MicroRNAs in Host Serum As Novel Biomarkers of Helminth Infection. PLoS Negl. Trop. Dis. 8, (2014).

17. Zhu, L. et al. Molecular characterization of S. japonicum exosome-like vesicles reveals their regulatory roles in parasite-host interactions. Sci. Rep. 6, 25885 (2016).

18. Liu, J. et al. Schistosoma japonicum extracellular vesicle miRNA cargo regulates host macrophage functions facilitating parasitism. PLOS Pathog. 15, e1007817 (2019).

19. van Niel, G., D’Angelo, G. \& Raposo, G. Shedding light on the cell biology of extracellular vesicles. Nat. Rev. Mol. Cell Biol. 19, 213 (2018).

20. Valadi, H. et al. Exosome-mediated transfer of mRNAs and microRNAs is a novel mechanism of genetic exchange between cells. Nat. Cell Biol. 9, 654 (2007).

21. Yáñez-Mó, M. et al. Biological properties of extracellular vesicles and their physiological functions. J. Extracell. Vesicles 4, 27066 (2015).

22. Zhu, S. et al. Release of extracellular vesicles containing small RNAs from the eggs of Schistosoma japonicum. Parasites and Vectors 9, 1-9 (2016).

23. Nowacki, F. C. et al. Protein and small non-coding RNA-enriched extracellular vesicles are released by the pathogenic blood fluke Schistosoma mansoni. J. Extracell. Vesicles 4, 28665 (2015).

24. Samoil, V. et al. Vesicle-based secretion in schistosomes: Analysis of protein and microRNA (miRNA) content of exosome-like vesicles derived from Schistosoma mansoni. Sci. Rep. 8, 3286 (2018).

25. Kifle, D. W. et al. Proteomic analysis of two populations of Schistosoma mansoni-derived extracellular vesicles: 15k pellet and 120k pellet vesicles. Mol. Biochem. Parasitol. 236, 111264 (2020).

26. Du, P. et al. Proteomic and deep sequencing analysis of extracellular vesicles isolated from adult male and female Schistosoma japonicum. PLoS Negl. Trop. Dis. 14, e0008618 (2020).

27. Wang, L. et al. Exosome-like vesicles derived by Schistosoma japonicum adult worms mediates M1 type immune- activity of macrophage. Parasitol. Res. 114, 1865-1873 (2015).

28. Torre-Escudero, E. de la \& Robinson, M. W. Extracellular vesicle-mediated communication in host-parasite interactions: insight from Fasciola hepatica . Ann. Transl. Med. Vol 5, Suppl. 1 (May 2017) Ann. Transl. Med. (2017). 
29. Fromm, B. et al. The revised microRNA complement of Fasciola hepatica reveals a plethora of overlooked microRNAs and evidence for enrichment of immuno-regulatory microRNAs in extracellular vesicles. Int. J. Parasitol. 45, 697-702 (2015).

30. Roig, J. et al. Extracellular Vesicles From the Helminth Fasciola hepatica Prevent DSS-Induced Acute Ulcerative Colitis in a T-Lymphocyte Independent Mode. Frontiers in Microbiology 9, 1036 (2018).

31. Coakley, G. et al. Extracellular Vesicles from a Helminth Parasite Suppress Macrophage Activation and Constitute an Effective Vaccine for Protective Immunity. Cell Rep. 19, 1545-1557 (2017).

32. Marco, A. et al. Sex-Biased Expression of MicroRNAs in Schistosoma mansoni. PLoS Negl. Trop. Dis. 7, e2402 (2013).

33. Catalano, M. \& O’Driscoll, L. Inhibiting extracellular vesicles formation and release: a review of EV inhibitors. J. Extracell. Vesicles 9, (2020).

34. Kosaka, N. et al. Secretory mechanisms and intercellular transfer of microRNAs in living cells. J. Biol. Chem. 285, 17442-17452 (2010).

35. Kalluri, R. \& LeBleu, V. S. The biology, function, and biomedical applications of exosomes. Science (80-. ). 367, (2020).

36. Datta, A. et al. Manumycin A suppresses exosome biogenesis and secretion via targeted inhibition of Ras/Raf/ERK1/2 signaling and hnRNP H1 in castration-resistant prostate cancer cells. Cancer Lett. 408, 73-81 (2017).

37. Jorfi, S. et al. Inhibition of microvesiculation sensitizes prostate cancer cells to chemotherapy and reduces docetaxel dose required to limit tumor growth in vivo. Sci. Rep. 5, 13006 (2015).

38. Shi, Z., Wang, Q., Zhang, Y. \& Jiang, D. Extracellular vesicles produced by bone marrow mesenchymal stem cells attenuate renal fibrosis, in part by inhibiting the RhoA/ROCK pathway, in a UUO rat model. Stem Cell Res. Ther. 11, 253 (2020).

39. Sotillo, J. et al. Extracellular vesicles secreted by Schistosoma mansoni contain protein vaccine candidates. Int. J. Parasitol. 46, 1-5 (2016).

40. AU - Liu, J. et al. Isolation and Characterization of Extracellular Vesicles from Adult Schistosoma japonicum. JoVE 57514 (2018). doi:doi:10.3791/57514

41. LoVerde, P. T. PRESIDENTIAL ADDRESS SEX AND SCHISTOSOMES: AN INTERESTING BIOLOGICAL INTERPLAY WITH CONTROL IMPLICATIONS. J. Parasitol. 88, 3-13 (2002).

42. Popiel, I. Male-stimulated female maturation inSchistosoma: A review. J. Chem. Ecol. 12, 1745-1754 (1986).

43. Popiel, I. \& Basch, P. F. Reproductive development of female Schistosoma mansoni (Digenea: Schistosomatidae) following bisexual pairing of worms and worm segments. J. Exp. Zool. 232, 141-150 (1984).

44. Lerman, G. et al. Schistosomal MicroRNAs Isolated From Extracellular Vesicles in Sera of Infected Patients: A New Tool for Diagnosis and Follow-up of Human Schistosomiasis. J. Infect. Dis. 215, 378-386 (2016).

45. Samoil, V. et al. Vesicle-based secretion in schistosomes: Analysis of protein and microRNA (miRNA) content of exosome-like vesicles derived from Schistosoma mansoni. Sci. Rep. 8, 1-16 (2018).

46. Chaimon, S. et al. Molecular characterization and functional analysis of the Schistosoma mekongi $\mathrm{Ca}(2+)$ dependent cysteine protease (calpain). Parasit. Vectors 12, 383 (2019).

47. Wang, Q., Da'dara, A. A. \& Skelly, P. J. The human blood parasite Schistosoma mansoni expresses extracellular tegumental calpains that cleave the blood clotting protein fibronectin. Sci. Rep. 7, 12912 (2017). 
48. Bennett, A. P. S., de la Torre-Escudero, E., Oliver, N. A. M., Huson, K. M. \& Robinson, M. W. The cellular and molecular origins of extracellular vesicles released by the helminth pathogen, Fasciola hepatica. Int. J. Parasitol. 50, 671-683 (2020).

49. Ohta, N. et al. Research on calpain of Schistosoma japonicum as a vaccine candidate. Parasitol. Int. 53, 175181 (2004).

50. Karmakar, S. et al. Cross-species protection: Schistosoma mansoni Sm-p80 vaccine confers protection against Schistosoma haematobium in hamsters and baboons. Vaccine 32, 1296-1303 (2014).

51. Le, L. et al. Schistosoma egg-induced liver pathology resolution by Sm-p80-based schistosomiasis vaccine in baboons. Pathology 50, 442-449 (2018).

52. Eyayu, T., Zeleke, A. J. \& Worku, L. Current status and future prospects of protein vaccine candidates against Schistosoma mansoni infection. Parasite Epidemiol. Control 11, e00176 (2020).

53. Kumagai, T. et al. Schistosoma japonicum: localization of calpain in the penetration glands and secretions of cercariae. Exp. Parasitol. 109, 53-57 (2005).

54. Molehin, A. J. et al. Cross-species prophylactic efficacy of Sm-p80-based vaccine and intracellular localization of Sm-p80/Sm-p80 ortholog proteins during development in Schistosoma mansoni, Schistosoma japonicum, and Schistosoma haematobium. Parasitol. Res. 116, 3175-3188 (2017).

55. Kumagai, T., Osada, Y., Ohta, N. \& Kanazawa, T. Peroxiredoxin-1 from Schistosoma japonicum functions as a scavenger against hydrogen peroxide but not nitric oxide. Mol. Biochem. Parasitol. 164, 26-31 (2009).

56. Ichimura, K., Kurihara, H. \& Sakai, T. Primary cilia disappear in rat podocytes during glomerular development. Cell Tissue Res. 341, 197-209 (2010).

\section{Tables}

Table1 


\begin{tabular}{|c|c|c|c|c|c|c|}
\hline $\begin{array}{l}\text { schisto- } \\
\text { miRNA }\end{array}$ & S1 & S2 & S3 & mean & S.D. & $\begin{array}{l}\text { miRBase } \\
\text { accession NO. }\end{array}$ \\
\hline $\begin{array}{l}\text { miR- } \\
125 b^{1}\end{array}$ & 33379.81067 & 37212.3302 & 30579.45579 & 33723.86555 & 3329.795197 & MIMAT0010179 \\
\hline miR-10 1 & 9429.853881 & 11641.73146 & 11910.93001 & 10994.17178 & 1361.409137 & MIMAT0016253 \\
\hline miR-277¹ & 2709.863774 & 3706.646173 & 3057.62312 & 3158.044356 & 505.9220354 & MIMAT0016269 \\
\hline miR-612 & 3194.070617 & 3234.35769 & 2534.474161 & 2987.634156 & 392.9646905 & MIMAT0016259 \\
\hline $\begin{array}{l}\text { miR- } \\
\text { bantam } 1,3\end{array}$ & 1844.383758 & 3458.624854 & 3401.288044 & 2901.432219 & 915.8796119 & MIMAT0010177 \\
\hline miR-36 & 1887.604804 & 2053.786193 & 2153.207223 & 2031.53274 & 134.1922996 & MIMAT0016257 \\
\hline $\begin{array}{l}\text { miR- } \\
3479^{1}\end{array}$ & 1687.738986 & 2122.004531 & 1976.619855 & 1928.787791 & 221.0487968 & MIMAT0016275 \\
\hline $\operatorname{miR}-71 a^{1}$ & 1489.940919 & 1875.130997 & 1504.408508 & 1623.160142 & 218.3330296 & MIMAT0010176 \\
\hline miR-3487 & 1011.634732 & 1884.263371 & 1611.475873 & 1502.457992 & 446.4122076 & MIMAT0016296 \\
\hline $\begin{array}{l}\text { miR- } \\
2162^{1}\end{array}$ & 1236.111835 & 1544.020042 & 1101.662512 & 1293.931463 & 226.7760527 & MIMAT0016273 \\
\hline $\begin{array}{l}\text { miR- } \\
125 a^{1}\end{array}$ & 736.5229389 & 798.1595521 & 816.0402324 & 783.5742411 & 41.71688462 & MIMAT0010178 \\
\hline let-7 $7^{1.2}$ & 384.2487176 & 339.8441009 & 335.794735 & 353.2958512 & 26.88232312 & MIMAT0010175 \\
\hline $\operatorname{miR}-2 a^{1}$ & 385.8625724 & 340.7423672 & 269.6632849 & 332.0894082 & 58.58091734 & MIMAT0016246 \\
\hline miR-3492 & 194.1668936 & 383.7094408 & 339.4565591 & 305.7776312 & 99.15793936 & MIMAT0016301 \\
\hline miR-3491 & 213.0792534 & 369.8861198 & 280.3754868 & 287.7802867 & 78.66525046 & MIMAT0016300 \\
\hline miR-3496 & 63.74726067 & 345.8325432 & 225.7213957 & 211.7670665 & 141.5594191 & MIMAT0016305 \\
\hline miR-2b & 50.53382531 & 369.2373718 & 205.7179984 & 208.4963985 & 159.3699384 & MIMAT0016247 \\
\hline miR-3498 & 56.88837819 & 304.0132544 & 227.9075594 & 196.2697307 & 126.5637794 & MIMAT0016307 \\
\hline miR-307 & 218.9294767 & 192.5284201 & 175.986173 & 195.8146899 & 21.6594439 & MIMAT0016270 \\
\hline
\end{tabular}

List of abundant miRNA contents of EVs of paired adult worms of S. japonicum. Only the top 20 miRNAs are shown. A complete list of all miRNAs that were detected is provided in Table S2. The means were calculated using analysis results of three individual miRNA libraries extracted from 5 paired adult worms.

${ }^{1}$ These miRNAs were detected in the serum of the infected animal ${ }^{16}$.

${ }^{2}$ This miRNA was male-biase (More than double the expression level) ${ }^{32}$.

${ }^{3}$ These miRNA were female-biased (More than double the expression level) ${ }^{32}$. 


\section{Figures}

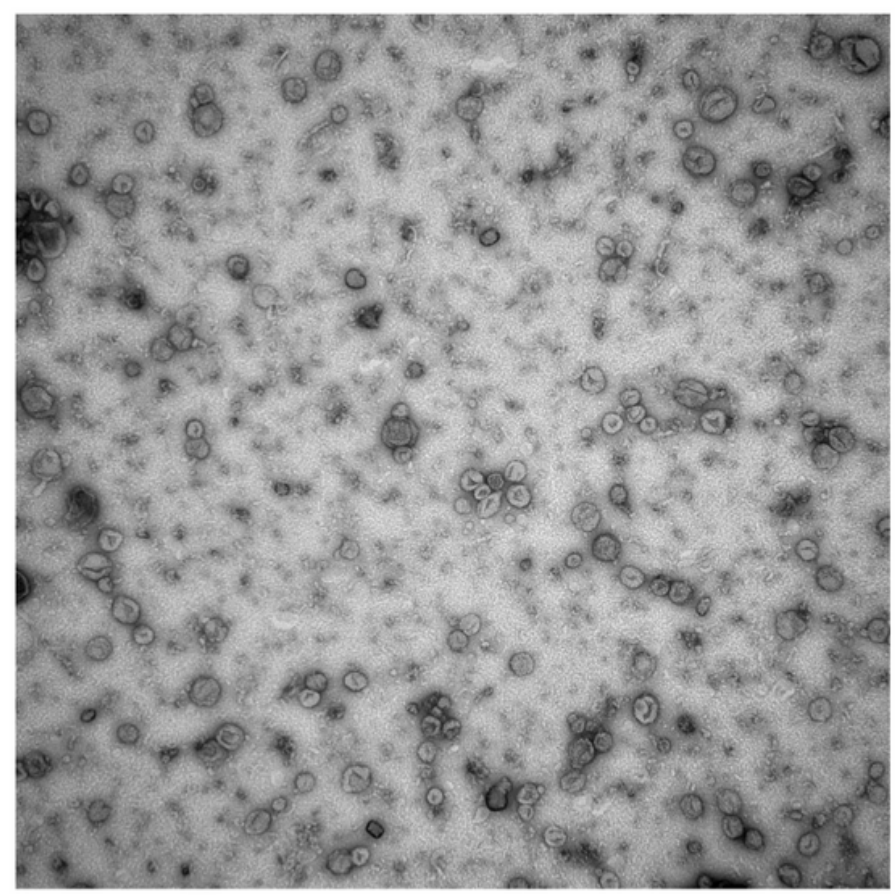

$500 \mathrm{~nm}$

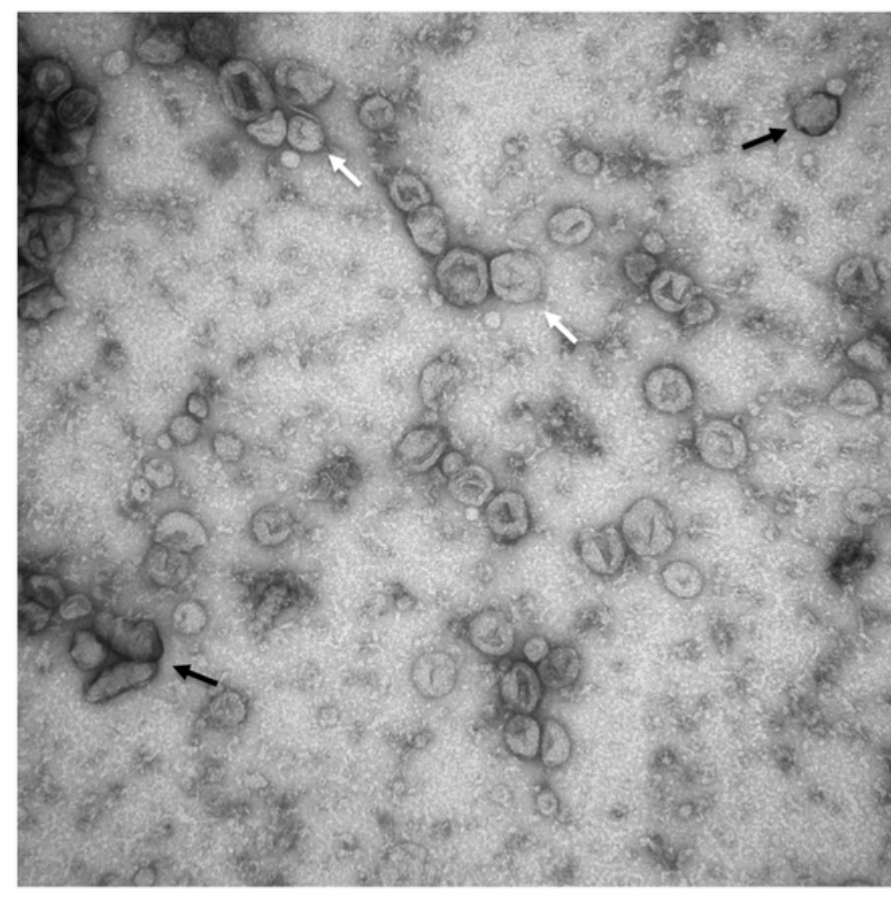

$100 \mathrm{~nm}$

\section{Figure 1}

Extracellular vesicles (EVs) from paired worms. Transmission electron micrographs showing EVs released from paired adult worms of Schistosoma japonicum. Two pictures were deposited onto a carbon-formvar coated grid, negatively stained in aqueous uranyl acetate, and analyzed on an $\mathrm{H}-7100$ transmission electron microscope. White arrows may indicate exosome-like, black arrows may indicate microvesicles (right panel). The scale bar indicates $500 \mathrm{~nm}$ in left panel, and $100 \mathrm{~nm}$ in right panel. 
$\mathbf{A}$

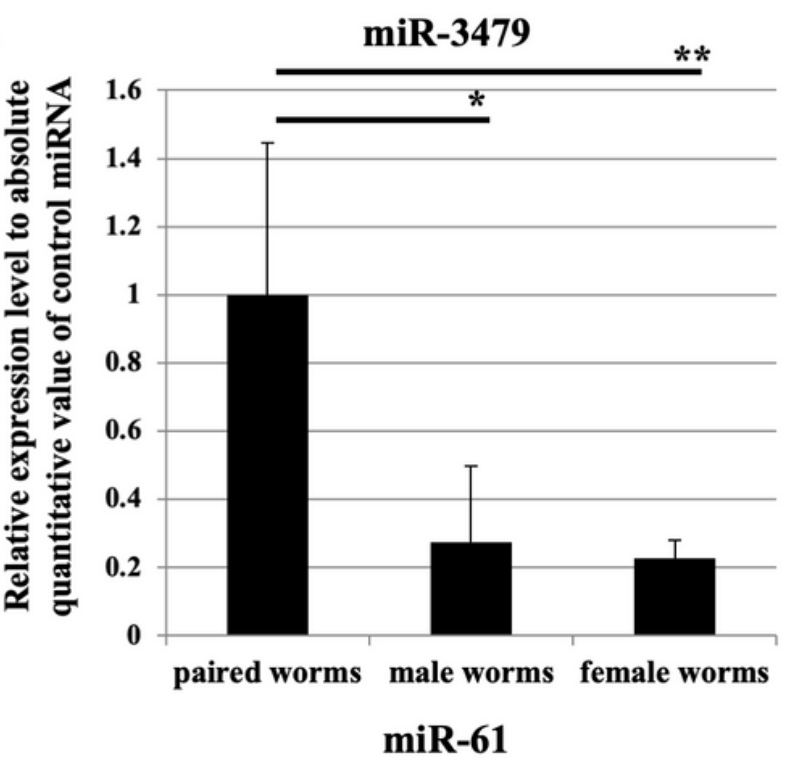

C

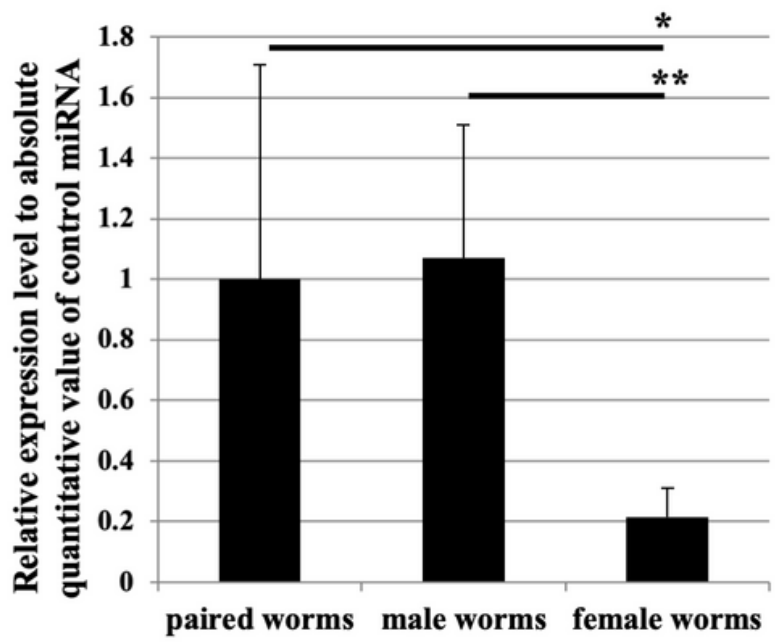

B

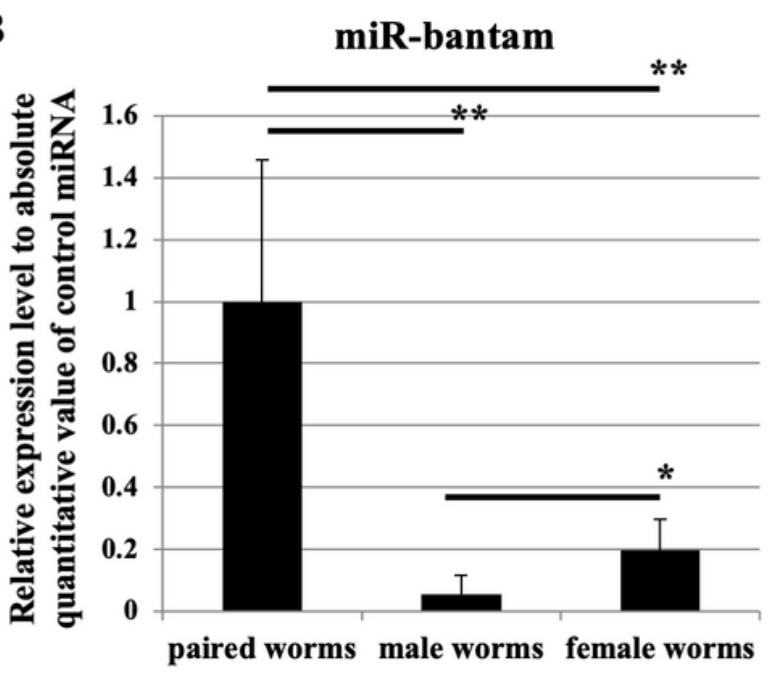

miR-36

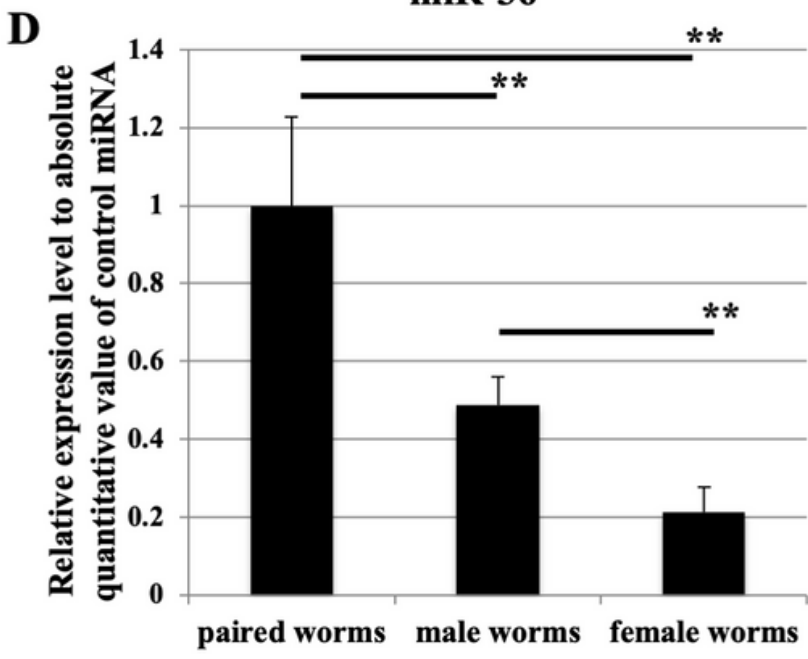

${ }^{*} \mathbf{p}<0.05$

$* * \mathbf{p}<0.01$

\section{Figure 2}

miRNA expression levels in EVs in the paired worms. Absolute quantification qRT-PCR analyses of enriched miRNAs (A) miR-3479, (B) miR-bantam, (C) miR-61, (D) miR-36 compared with paired worm group. * means $\mathrm{P} \leq 0.05$ and ** means $P \leq 0.01$. Data illustrate representative findings and show the mean and standard errors derived from 5 groups. 
A

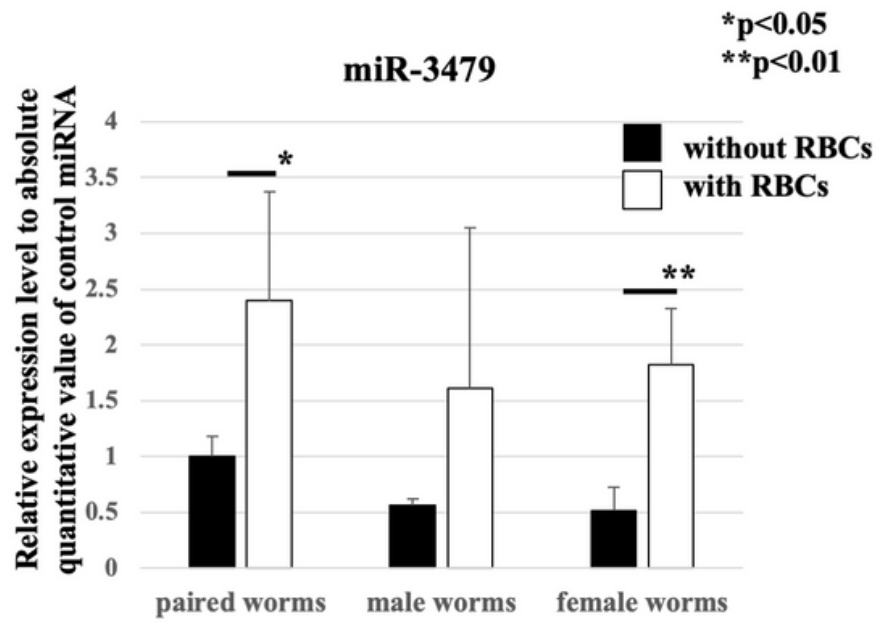

B

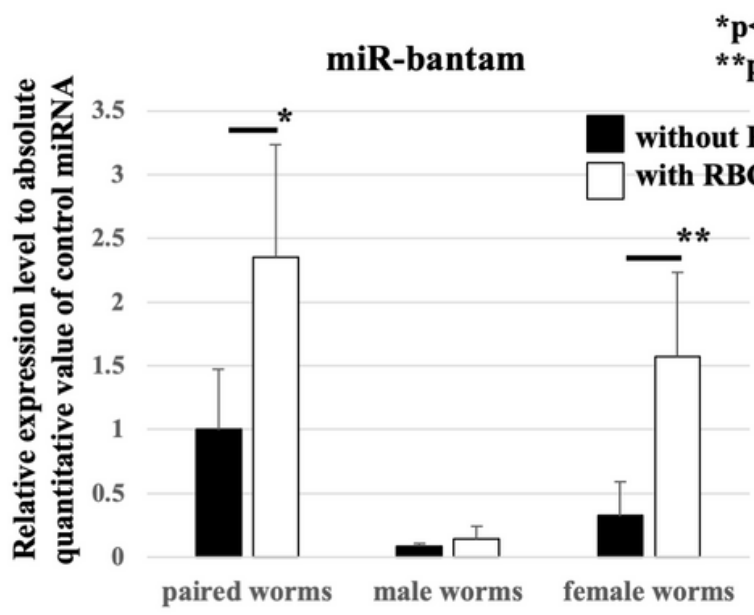

C

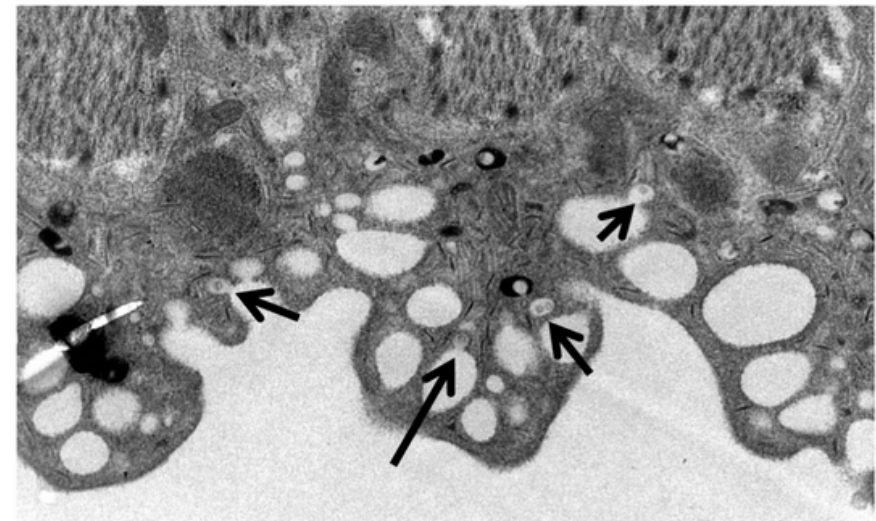

$500 \mathrm{~nm}$

\section{Figure 3}

miRNA expression levels in EVs released from adult worms with erythrocytes, and ultrastructural observation by TEM of the surface of female worms treated with the erythrocytes. Absolute quantification qRT-PCR analyses of miRNAs (A) miR-3479, (B) miR-bantam compared with paired worm group. * means $P \leq 0.05$ and ** means $P \leq 0.01$. Data illustrate representative findings and show the mean and standard errors derived from 5 groups. (C) Transmission electron micrographs showing EVs in the tegument (medial posterior portion ) of female worms cultured with RBCs. The EVs were frequently found in the vacuolar invaginations of tegmental epithelial cells (arrows). 
$\mathbf{A}$

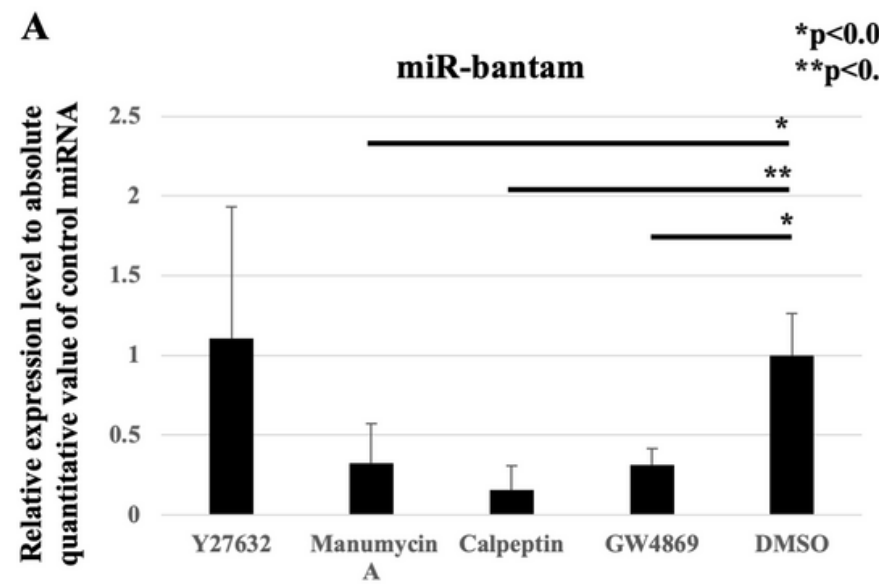

C
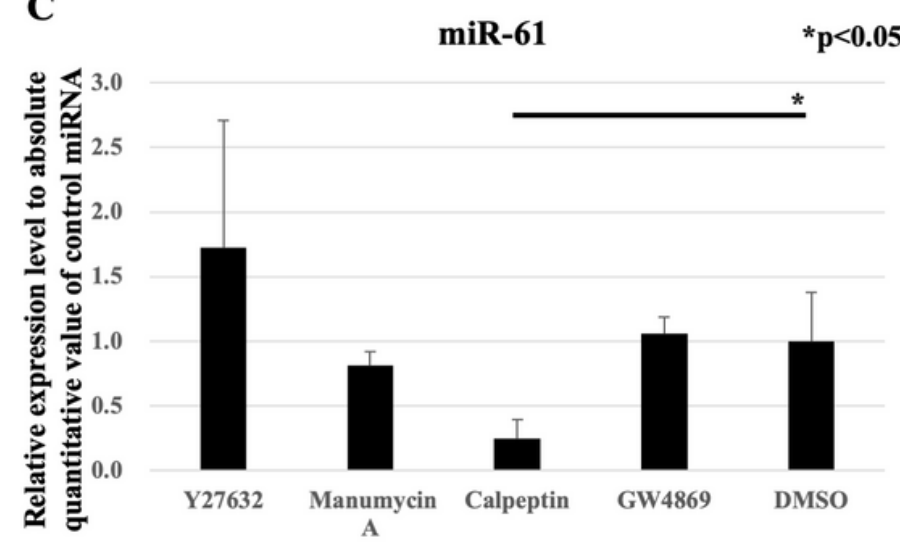

B

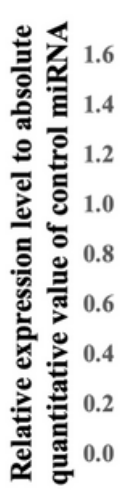

D

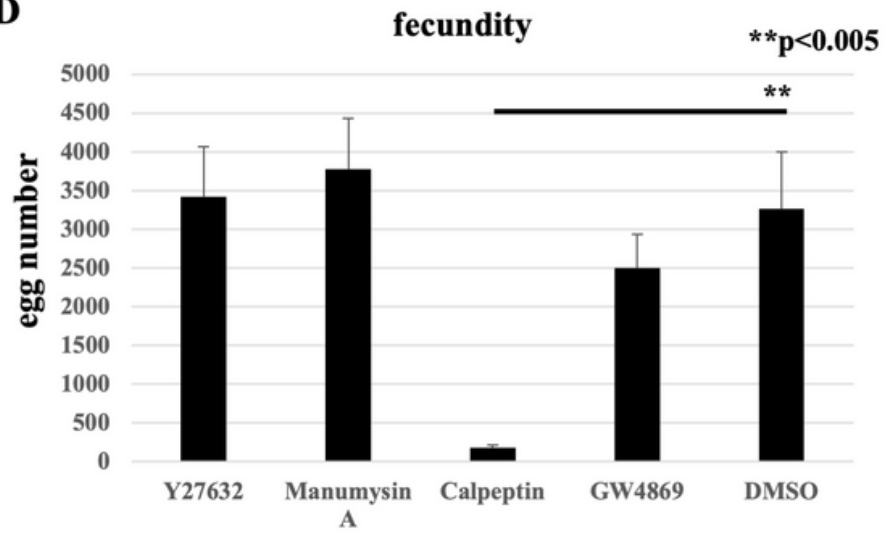

Figure 4

miRNA expression levels in EV after treatment with inhibitors paired adults with inhibitors. Absolute quantification qRT-PCR analyses of miRNAs (A) miR- bantam, (B) miR-3479, (C) miR-61 compared with DMSO-treated group. * means $P \leq 0.05$ and $* *$ means $P \leq 0.01$. Data illustrate representative findings and show the mean and standard errors derived from triplicate. (D) Fecundity of paired worms cultured with each inhibitor in vitro. Egg numbers were counted under microscopy after dissolving $4 \% \mathrm{KOH}$. Data show the mean and standard errors derived from triplicate groups. This experiment was repeated twice (the first experiment is shown).

\section{Supplementary Files}

This is a list of supplementary files associated with this preprint. Click to download.

- Supplementaryinformation.pdf 